\section{Experimental Confirmation of 3- and 5-Scroll Attractors from a Generalized Chua's Circuit}

\author{
M. E. Yalçin, J. A. K. Suykens, and J. Vandewalle
}

Abstract-In this letter we present an experimental confirmation of 3and 5-scroll attractors from a generalized Chua's circuit. The generalized Chua's circuit introduced by Suykens, Huang, and Chua makes use of a piecewise linear characteristic with multiple breakpoints leading to a more complete family of $n$-scroll attractors than $n$-double scroll attractors, with $n$ a natural number.

Index Terms-Chua's circuits, $n$-(double) scrolls.

\section{INTRODUCTION}

Chua's circuit is a paradigm for chaos [12], being a simple nonlinear electrical circuit that exhibits a rich variety of phenomena [3]-[7], which has been experimentally confirmed in [23]. Among the generalizations that have been proposed in literature [8], [10]-[12], [21], Suykens and Vandewalle [13], [14] introduced a family of $n$-double scroll attractors, where $n$ is a natural number. An experimental confirmation of 2-double scrolls has been given by Arena et al. in [1], [2]. In this work a piecewise linear characteristic with multiple breakpoints has been taken. A more complete family of $n$-scroll instead of $n$-double scroll attractors has been obtained from the generalized Chua's circuit proposed by Suykens, Huang and Chua in [15]. In this circuit an additional scroll around the origin can be realized, leading in this way to a family with an even or odd number of scrolls. The generalized Chua's circuit is representable in Lur'e form and as a result can and has been used within synchronization schemes for secure communications using chaos [17], [18], [20] Arrays of cells consisting of generalized Chua's circuits leading to hyperchaotic behavior with $n$-double scroll hypercube attractors have been studied in [16]. Finally, a time-series prediction competition has been organized at the K.U. Leuven Belgium, where the behavior of a simulated 5-scroll attractor (after a nonlinear transformation) had to be predicted, given 2000 data points of the attractor. The results of this contest have been reported in [19]. In this paper we experimentally confirm the 3-scroll and 5-scroll attractor from the generalized Chua's circuit. The circuit implementation is based on the realization of Chua's circuits by a voltage controlled voltage source [22].

This paper is organized as follows. In Section II we give the equations of the generalized Chua's circuit. In Section III the realization of 3- and 5-scroll attractors is discussed.

\section{Generalized ChuA's Circuit}

The generalized Chua's circuit, which exhibits an even or odd number of scrolls, as proposed by Suykens, Huang and Chua, is given by the equations [1], [12], [15]

$$
\left\{\begin{array}{l}
\dot{x}=\alpha[y-h(x)] \\
\dot{y}=x-y+z \\
\dot{z}=-\beta y
\end{array}\right.
$$

Manucript received October 2, 1998; revised March 9, 1999. This paper was recommended by Associate Editor C. W. Wu.

The authors are with the Katholieke Universiteit Leuven, Department of Electrical Engineering, ESAT-SISTA, B-3001 Leuven (Heverlee), Belgium (e-mail johan.suykens@esat.kuleuven.ac.be).

Publisher Item Identifier S 1057-7122(00)02318-7.
TABLE I

COMPONENT LIST

\begin{tabular}{llll}
\hline Element & Description & Value & Tolerance \\
\hline \hline & Op amp (TL082) & & \\
R11 & $\frac{1}{4}$ W Resistor & $13 k \Omega$ & $\mp \% 1$ \\
R12 & $\frac{1}{4}$ W Resistor & $11 k \Omega$ & $\mp \% 1$ \\
R21 & $\frac{1}{4}$ W Resistor & $5.1 k \Omega$ & $\mp \% 1$ \\
R22 & $\frac{1}{4}$ W Resistor & $24 k \Omega$ & $\mp \% 1$ \\
R31 & $\frac{1}{4}$ W Resistor & $1.3 k \Omega$ & $\mp \% 1$ \\
R32 & $\frac{1}{4}$ W Resistor & $13 k \Omega$ & $\mp \% 1$ \\
R41 & $\frac{1}{4}$ W Resistor & $1.3 k \Omega$ & $\mp \% 1$ \\
R42 & $\frac{1}{4}$ W Resistor & $8.2 k \Omega$ & $\mp \% 1$ \\
R51 & $\frac{1}{4}$ W Resistor & $24 k \Omega$ & $\mp \% 1$ \\
R52 & $\frac{1}{4}$ W Resistor & $1.5 k \Omega$ & $\mp \% 1$ \\
R61 & $\frac{1}{4}$ W Resistor & $1.2 k \Omega$ & $\mp \% 1$ \\
R62 & $\frac{1}{4}$ W Resistor & $20 k \Omega$ & $\mp \% 1$ \\
R71 & $\frac{1}{4}$ W Resistor & $68 k \Omega$ & $\mp \% 1$ \\
R72 & $\frac{1}{4}$ W Resistor & $1.8 k \Omega$ & $\mp \% 1$ \\
R81 & $\frac{1}{4}$ W Resistor & $1.1 k \Omega$ & $\mp \% 1$ \\
R82 & $\frac{1}{4}$ W Resistor & $30 k \Omega$ & $\mp \% 1$ \\
R91 & $\frac{1}{4}$ W Resistor & $130 k \Omega+11 k \Omega$ & $\mp \% 1$ \\
R92 & $\frac{1}{4}$ W Resistor & $2.2 k \Omega$ & $\mp \% 1$ \\
R1 & $\frac{1}{4}$ W Resistor & $100 k \Omega$ & $\mp \% 1$ \\
R2 & $\frac{1}{4}$ W Resistor & $100 k \Omega$ & $\mp \% 1$ \\
\hline R3 & $\frac{1}{4}$ W Resistor & $5.1 k \Omega$ & $\mp \% 1$ \\
\hline R & $\frac{1}{4}$ W Resistor & $10 k \Omega$ & \\
$R$ G & $\frac{1}{4}$ W Resistor & $10 k \Omega$ & \\
C1 & Capacitor & $1 n F$ & \\
C2 & Capacitor & $20 n F$ & \\
L & Ferroxcube self & $68 m H+1$ \\
& & & \\
\hline
\end{tabular}

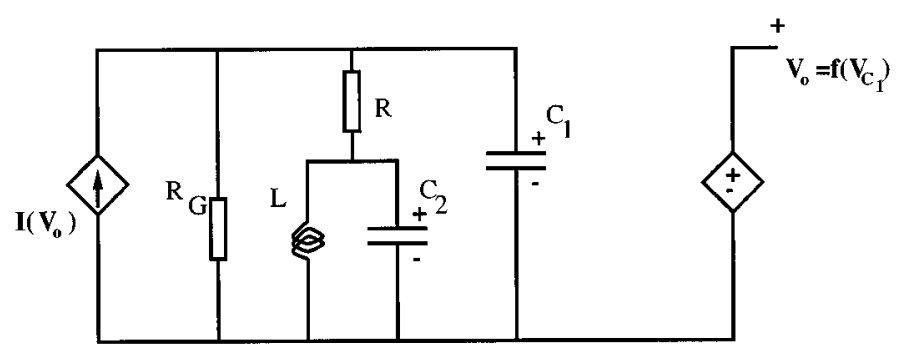

Fig. 1. Voltage controlled voltage source (VCVS) implementation of Chua's circuit.

having a piecewise linear characteristic with multiple breakpoints

$$
\begin{aligned}
h(x)= & m_{2 q-1}+\frac{1}{2} \sum_{i=1}^{2 q-1}\left(m_{i-1}-m_{i}\right) \\
& \times\left(\left|x+c_{i}\right|-\left|x-c_{i}\right|\right)
\end{aligned}
$$

where $q$ denotes a natural number. Existing circuit implementations of (1), (2) have been made for $q=1$ corresponding to the double scroll case [12] and by Arena et al. in [1], [2] in order to confirm the $n$-double scroll attractors, introduced by Suykens and Vandewalle in [13], [14]. 


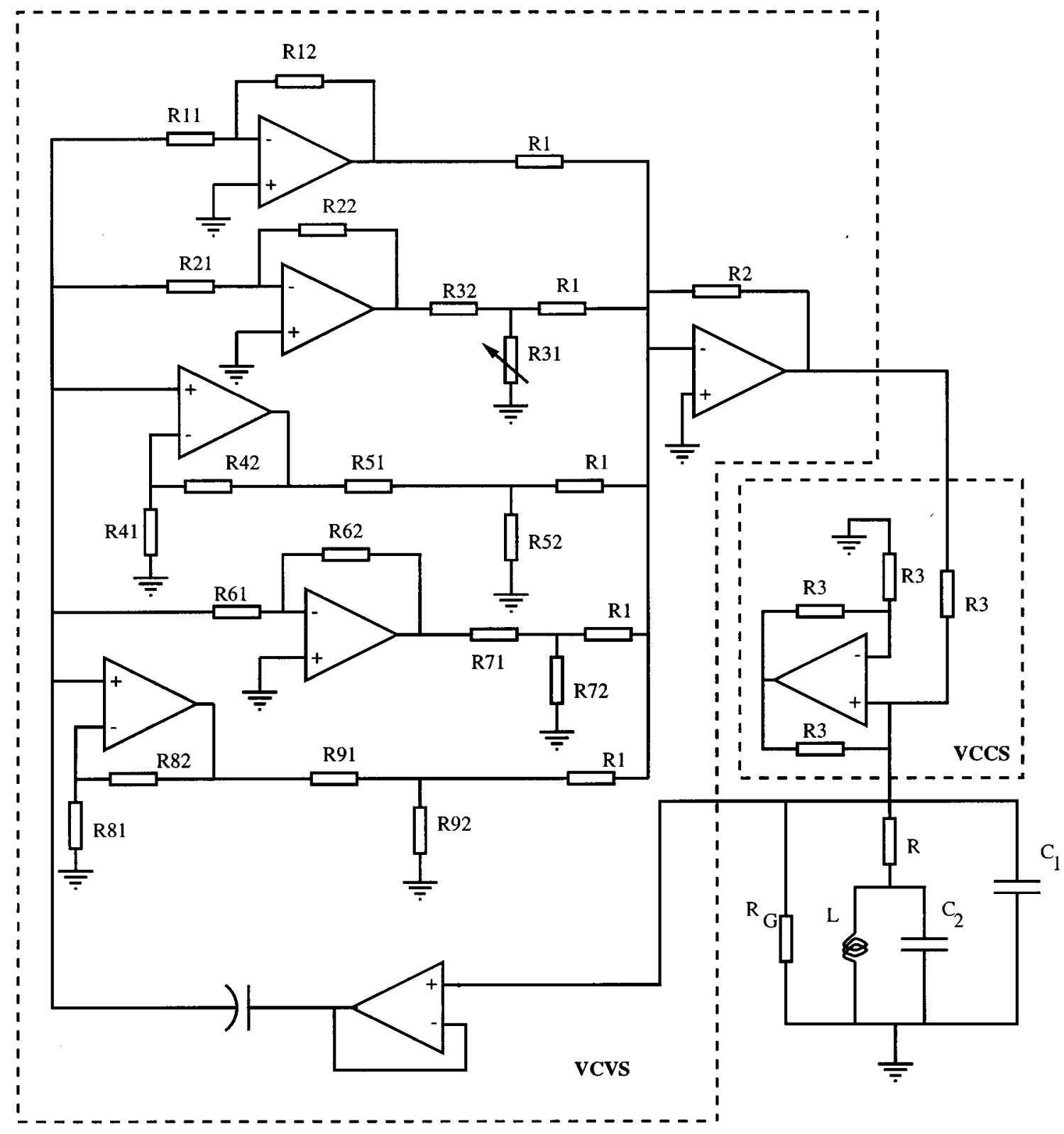

Fig. 2. Realization of the 5-scroll generating generalized Chua's circuit.

Taking $q=n$ one obtains the $n$-double scroll attractors for the parameters $\alpha=9, \beta=14.286$ with the vectors $m=\left[m_{0} ; m_{1} ; \ldots ; m_{2 q-1}\right]$, $c=\left[c_{1} ; c_{2} ; \ldots ; c_{2 q-1}\right]$ chosen as

- $q=1$ : double scroll (2-scroll) [3]-[7], [12], [23]

$$
\begin{aligned}
m & =[-1 / 7 ;+2 / 7] \\
c & =1
\end{aligned}
$$

- $q=2$ : 2-double scroll (4-scroll) [1], [2]

$$
\begin{aligned}
m & =[-1 / 7 ;+2 / 7 ;-4 / 7 ;+2 / 7] \\
c & =[1 ; 2.15 ; 3.6]
\end{aligned}
$$

- $q=2: 3$-scroll [15]

$$
\begin{aligned}
m & =[+0.9 / 7 ;-3 / 7 ;+3.5 / 7 ;-2.4 / 7] \\
c & =[1 ; 2.15 ; 4]
\end{aligned}
$$

- $q=3$ : 5-scroll [15]

$$
\begin{aligned}
m & =[+0.9 / 7 ;-3 / 7 ;+3.5 / 7 ;-2.7 / 7 ;+4 / 7 ;-2.4 / 7] \\
c & =[1 ; 2.15 ; 3.6 ; 6.2 ; 9] .
\end{aligned}
$$

The components of the vector $m$ have alternating signs and the sign of $m_{0}$ is negative in the case of $n$-double scrolls. $n$-Scroll attractors with an odd number of scrolls are generated from (1) and (2) by taking the same values for the $\alpha, \beta$ parameters but the opposite sign for the vector $m$ in the nonlinearity.

\section{REALIZATION OF 3- AND 5-SCROLl ATtRACTORS}

The proposed circuit model is shown in Fig. 1, in which a parallel combination of the linear resistor $R_{G}$ and a linear Voltage-Controlled Current Source (VCCS) is equivalent to Chua's diode. For this type of realization the corresponding state equations are given by

$$
\left\{\begin{array}{l}
\dot{x}=\alpha[-(1+\delta) x+y+f(x)] \\
\dot{y}=x-y+z \\
\dot{z}=-\beta y
\end{array}\right.
$$

with $f(x)=-h(x)+(1+\delta) x$ and $\delta=1$ [9]. The realization has been introduced in [22] for the generation of double scroll attractors from Chua's circuit. 


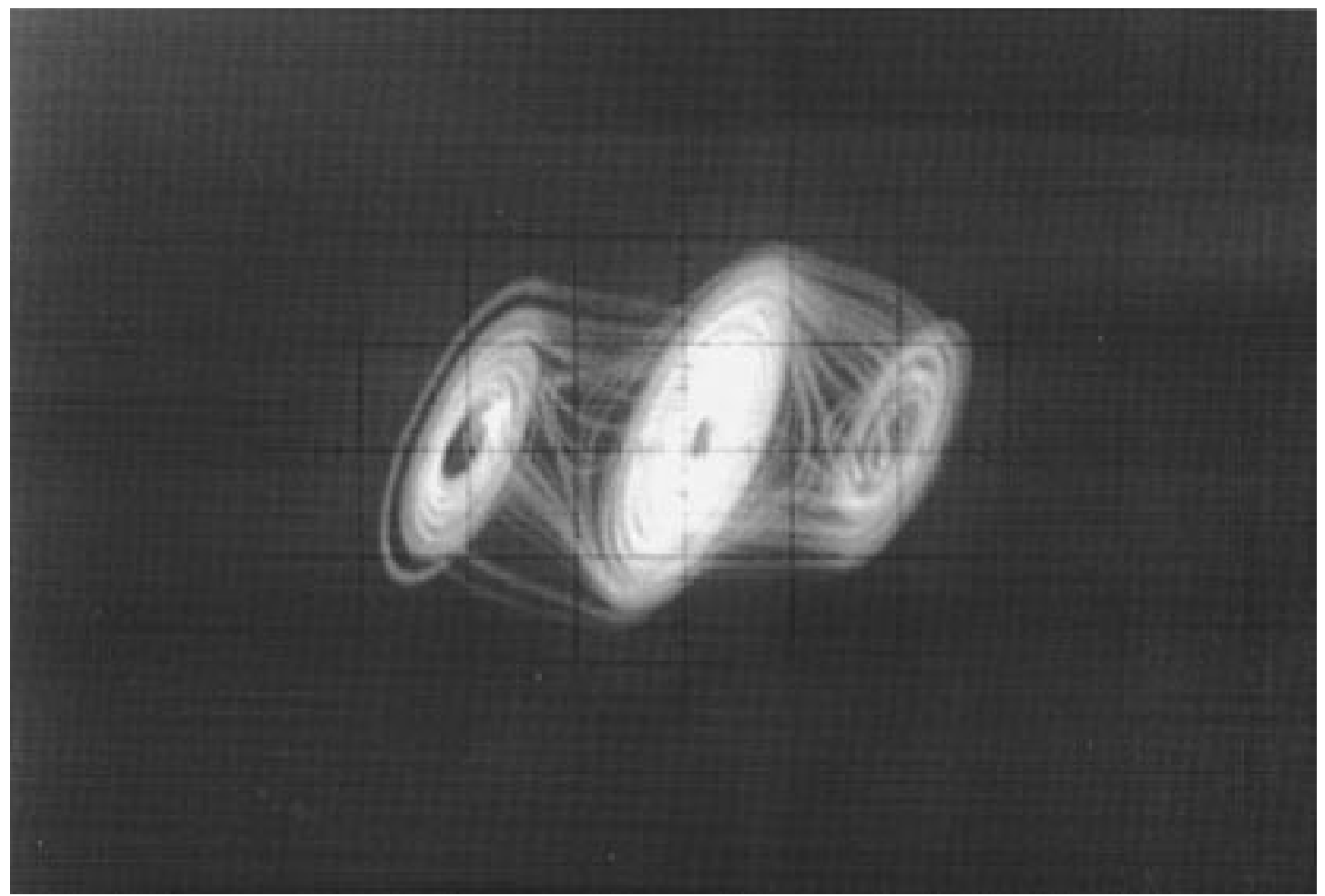

(a)

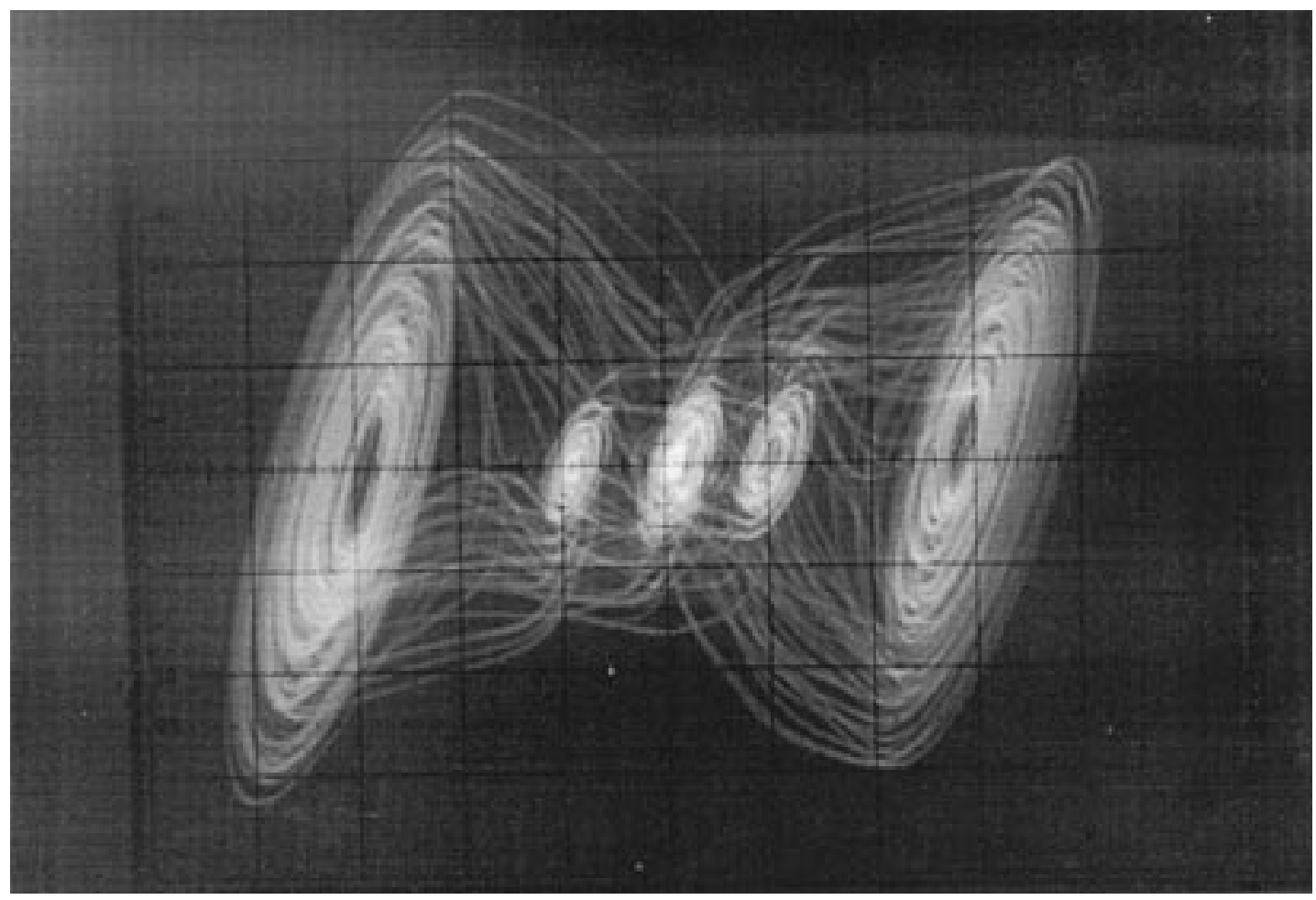

(b)

Fig. 3. Experimental confirmation of 3- and 5-scroll attractors: (a) 3-scroll $R_{31}=12280 \Omega$ (horizontal axis $V_{C 1} 0.5 \mathrm{~V} /$ div, vertical axis $V_{C 2} 0.1 \mathrm{~V} / \mathrm{div}$ ); (b) 5 -scroll $R_{31}=12740 \Omega$ (horizontal axis $V_{C 1} 1 \mathrm{~V} /$ div, vertical axis $V_{C 2} 0.2 \mathrm{~V} / \mathrm{div}$ ).

In our simulation, we have obtained that the last PWL term is not necessary in order to realize 5-scroll attractors. Hence $f(x)$ is obtained by the output of the linear VCCS which is controlled by a sum of five terms which are separately realized by inverting/noninverting amplifiers and voltage branches. The outputs of the each term are saturated when their input reaches the desired break-point. The break-points are adjusted with gains of inverting/noninverting amplifiers

$$
c_{4}=\frac{E_{\text {sat }}}{\frac{R_{22}}{R_{21}}}, \quad c_{3}=\frac{E_{\text {sat }}}{1+\frac{R_{42}}{R_{41}}}
$$




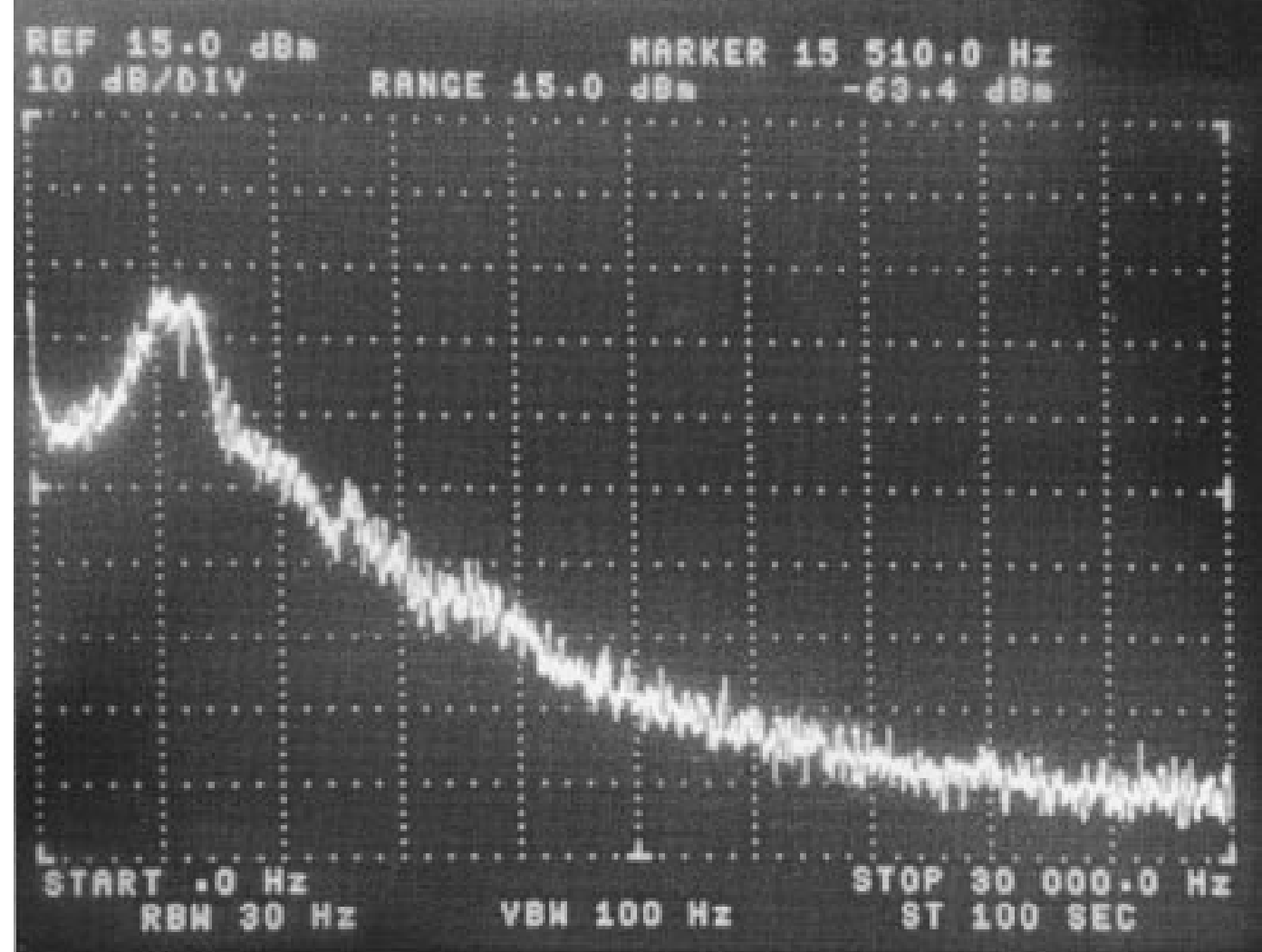

(a)

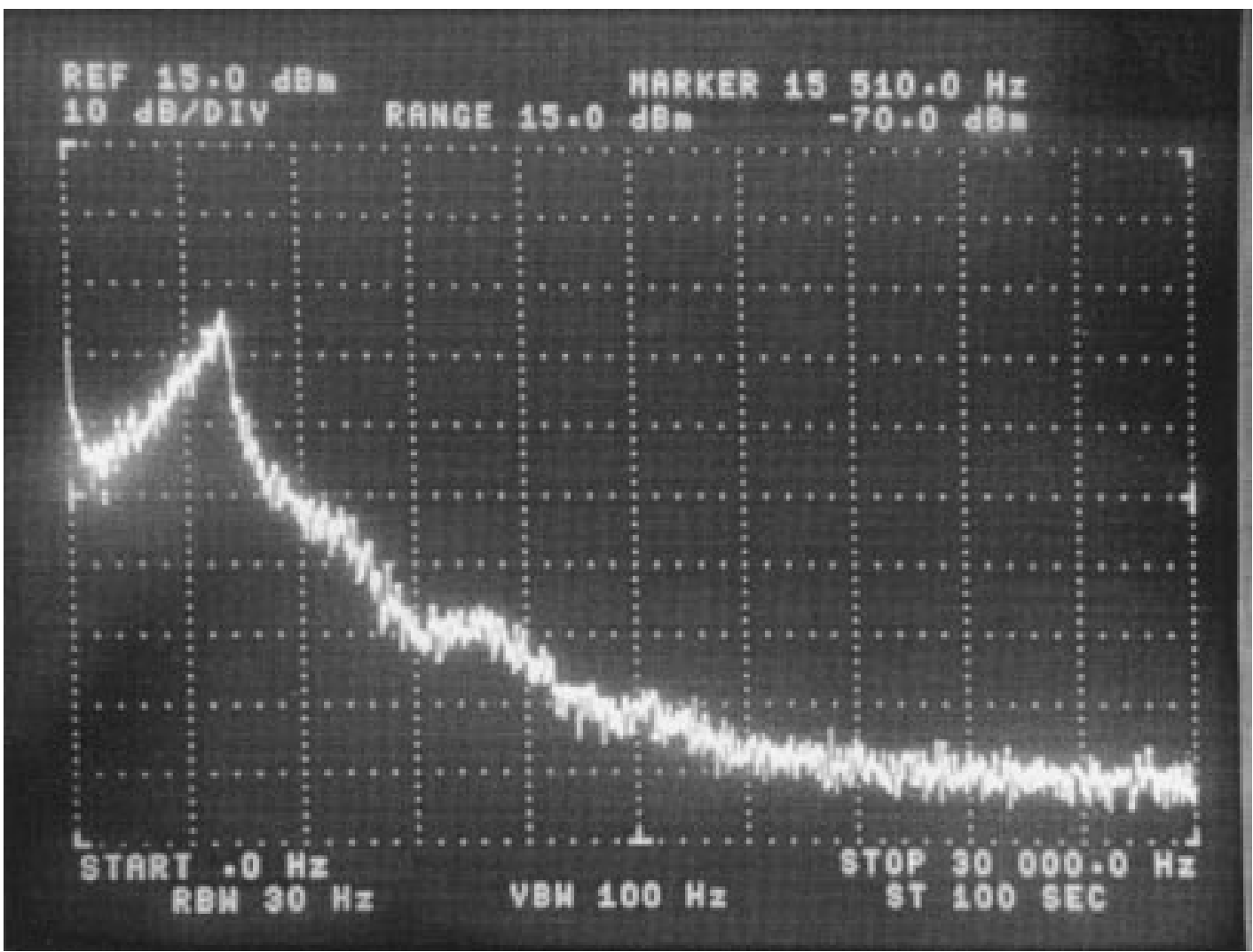

(b)

Fig. 4. Power spectra measured from $V_{C 1}$ : (a) 3-scroll; (b) 5-scroll attractors. 


$$
c_{2}=\frac{E_{\mathrm{sat}}}{\frac{R_{62}}{R_{61}}}, \quad c_{1}=\frac{E_{\mathrm{sat}}}{1+\frac{R_{82}}{R_{81}}} .
$$

The slopes are adjusted with the gains of inverting/noninverting amplifiers and voltage branches:

$$
\begin{aligned}
& m_{4}=-\frac{1}{R_{3}} \frac{R_{2}}{R_{1}}\left[-\frac{R_{12}}{R_{11}}\right] \\
& m_{3}=-\frac{1}{R_{3}} \frac{R_{2}}{R_{1}}\left[m_{4}+\left(-\frac{R_{22}}{R_{21}}\right) \frac{R_{31}}{R_{31}+R_{32}}\right] \\
& m_{2}=-\frac{1}{R_{3}} \frac{R_{2}}{R_{1}}\left[m_{3}+\left(1+\frac{R_{42}}{R_{41}}\right) \frac{R_{52}}{R_{52}+R_{51}}\right] \\
& m_{1}=-\frac{1}{R_{3}} \frac{R_{2}}{R_{1}}\left[m_{2}+\left(-\frac{R_{62}}{R_{61}}\right) \frac{R_{72}}{R_{72}+R_{71}}\right] \\
& m_{0}=-\frac{1}{R_{3}} \frac{R_{2}}{R_{1}}\left[m_{1}+\left(1+\frac{R_{82}}{R_{81}}\right) \frac{R_{92}}{R_{91}+R_{92}}\right] .
\end{aligned}
$$

The 5-scroll attractor has been obtained for $\alpha=18, \beta=28.56, m=$ $[1.7089 ; 2.5776 ; 1.7180 ; 2.5777 ; 1.6923] c=[0.5305 ; 0.9000$ $2.0526 ; 3.1875]$. The corresponding capacitor and resistor values for the 5-scroll attractor are given by $C_{1}=1 / 9 \mathrm{~F}, C_{2}=2 \mathrm{~F}, R=1$ $\Omega, R_{G}=1 \Omega, L=0.07 \mathrm{H}$. When current and time are rescaled by a factor 10000 , the parameters become $C_{1}=10 / 9 \mathrm{nF}, C_{2}=20$ $\mathrm{nF}, R=10 \mathrm{k} \Omega, R_{6}=10 \mathrm{k} \Omega, L=70 \mathrm{mH}$. This results into $m=[0.1649 \mathrm{mS} ; 0.2498 \mathrm{mS} ; 0.1655 \mathrm{mS} ; 0.2498 \mathrm{mS} ; 0.1659 \mathrm{mS}]$, with unchanged location of breakpoints. $C_{1}$ and $L$ are not standard values, therefore we have chosen $C_{1}=1 \mathrm{nF}$ and $L=70.2 \mathrm{mH}$. The implemented circuit has been shown in Fig. 2. The component list is given in Table I.

By adjusting the value of $R_{31}$ in Fig. 2 from $12280 \Omega$ to $12740 \Omega$ the generalized Chua's circuit behaves as a 3 -scroll instead of a 5-scroll, as illustrated in Fig. 3. A two dimensional view of the attractor is obtained by connecting $V_{C 1}$ and $V_{C 2}$ to the $X$ and $Y$ channels respectively of an $X-Y$ oscilloscope. Fig. 4 shows the spectrum of 3- and 5-scroll attractors for $V_{C 1}$.

\section{CONCLUSION}

A circuit implementation of 3- and 5-scroll attractors has been given. The attractors are generated from generalized Chua's circuits that have multiple breakpoints in the PWL characteristic. The generalized Chua's circuits are representable in Lur'e form. Hence they can be employed in methods for secure communications using chaos that exploit properties of Lur'e systems, besides other general methods. A computer simulated 5 -scroll attractor has been used in the K. U. Leuven time series prediction competition.

\section{ACKNOWLEDGMENT}

This research work was carried out at the ESAT laboratory and the Interdisciplinary Center of Neural Networks ICNN of the Katholieke Universiteit Leuven, in the framework of the the Concerted Action Project MIPS (Modelbased Information Processing Systems) of the Flemish Community and ESPRIT IV 27077 (DICTAM). J. Suykens is a postdoctoral researcher with the National Fund for Scientific Re- search FWO-Flanders. The authors would like to thank J. Bienstman and J. Schier for their kind assistance with taking the pictures.

\section{REFERENCES}

[1] P. Arena, S. Baglio, L. Fortuna, and G. Manganaro, "Generation of $n$-double scrolls via cellular neural networks," Int. J. Circuit Theory Appl., vol. 24, pp. 241-252, 1996.

[2] P. Arena, S. Baglio, L. Fortuna, and G. Manganaro, "State controlled CNN: A new strategy for generating high complex dynamics," IEICE Trans. Fundamentals, vol. E79-A, pp. 1647-1657, 1996.

[3] L. O. Chua, M. Komuro, and T. Matsumoto, "The double scroll family," IEEE Trans. Circuits Syst. I, vol. 33, pp. 1072-1118, Nov. 1986.

[4] L. O. Chua, "The genesis of Chua's circuit," Archiv für Elektronik und Übertragungstechnik, vol. 46, no. 4, pp. 250-257, 1992.

[5] L. O. Chua, "Global unfolding of Chua's circuit," IEICE Trans. Fundamentals, vol. E76-A, no. 5, pp. 704-734, 1993.

[6] L. O. Chua, C. W. Wu, A. Huang, and G.-Q. Zhong, "A universal circuit for studying and generating chaos-Part I: Routes to chaos \& Part II: Strange attractors," IEEE Trans. Circuits Syst. I, vol. 40, pp. 732-744 \& 745-761, Oct. 1993.

[7] L. O. Chua, "Chua's circuit: An overview ten years later," J. Circuits, Syst., Comp., vol. 4, no. 2, pp. 117-159, 1994.

[8] M. Götz, U. Feldmann, and W. Schwarz, "Synthesis of higher-dimensional Chua circuits," IEEE Trans. Circuits Syst. I, vol. 40, pp. 854-860, Nov. 1993.

[9] C. Güzeliş, "Chaotic cellular neural networks made of Chua's circuits," in Chua's Circuit: A Paradigm for Chaos. ser. B, R. N. Madan, Ed, Singapore: World Scientific, 1993, vol. 1, pp. 952-961.

[10] A. Huang, L. Pivka, C.-W. Wu, and M. Franz, "Chua's equation with cubic nonlinearity," Int. J. Bifurcation Chaos, vol. 6, no. 8, 1996.

[11] L. Kocarev, L. Karadzinov, and L. O. Chua, " $n$-dimensional canonical Chua's circuit," J. Circuits, Syst., Comp., vol. 3, no. 1, pp. 239-258, 1993.

[12] R. N. Madan, Ed., Chua's Circuit: A Paradigm for Chaos, Signapore: World Scientific , 1993.

[13] J. A. K. Suykens and J. Vandewalle, "Quasilinear approach to nonlinear systems and the design of $n$-double scroll $(n=1,2,3,4, \ldots)$," Inst. Elect. Eng., pt. G, vol. 138, no. 5, pp. 595-603, 1991.

[14] J. A. K. Suykens and J. Vandewalle, "Generation of $n$-double scrolls $(n=1,2,3,4, \ldots)$," IEEE Trans. Circuits Syst. I, vol. 40, pp. 861-867, Nov. 1993.

[15] J. A. K. Suykens, A. Huang, and L. O. Chua, "A family of $n$-scroll attractors from a generalized Chua's circuit," Archiv fur Elektronik und Ubertragungstechnik, vol. 51, no. 3, pp. 131-138, 1997.

[16] J. A. K. Suykens and L. O. Chua, "n-double scroll hypercubes in 1D-CNNs," Int. J. Bifurcation Chaos, vol. 7, no. 8, pp. 1873-1885, 1997.

[17] J. A. K. Suykens, J. Vandewalle, and L. O. Chua, "Nonlinear $H_{\infty}$ synchronization of chaotic Lur'e systems," Int. J. Bifurcation Chaos, vol. 7, no. 6, pp. 1323-1335, 1997.

[18] J. A. K. Suykens, P. F. Curran, J. Vandewalle, and L. O. Chua, "Robust nonlinear $H_{\infty}$ synchronization of chaotic Lur'e systems," IEEE Trans. Circuits Syst. I, vol. 44, pp. 891-904, Oct. 1997.

[19] J. A. K. Suykens and J. Vandewalle, "The K.U. Leuven time series prediction competition," in Nonlinear Modeling: Advanced Black-Box Techniques, J. A. K. Suykens and J. Vandewalle, Eds. Boston, MA: Kluwer , 1998, pp. 241-253.

[20] C. W. Wu and L. O. Chua, "A unified framework for synchronization and control of dynamical systems," in Int. J. Bifurcation Chaos, 1994, vol. 4, pp. 979-989.

[21] C. W. Wu and L. O. Chua, "On the generality of the unfolded Chua's circuit," in Int. J. Bifurcation Chaos, 1996, vol. 6, pp. 801-832.

[22] M. E. Yalçin, L. Şavkay, and F. A. Savaci, "New realization of Chua's circuit by using voltage controlled voltage source (VCVS)," in Int. Workshop Nonlinear Dynamics Electronic Systems NDES'96, June 1996, pp. 259-264.

[23] G.-Q. Zhong and F. Ayrom, "Experimental confirmation of chaos from Chua's circuit,” Int. J. Circuit Theory Appl., vol. 13, pp. 93-98, 1985. 